\title{
Peer-reviewed Brainstorming to Facilitate Large Group Collaboration
}

\author{
Joel H. Helquist \\ Utah Valley University \\ joel.helquist@uvu.edu
}

\author{
John Kruse \\ MITRE \\ john@kruser.org
}

\author{
Christopher B.R. Diller \\ University of Nebraska \\ cdiller@unomaha.edu
}

\begin{abstract}
This paper examines the impact of peer-reviewed brainstorming on the quality of brainstorming ideas. Peer-reviewed brainstorming aims to improve the quality of the brainstorming ideas and reduce the number of noisy comments. A pilot study was conducted that compared traditional, free brainstorming to a peer-reviewed brainstorming process, which requires each idea to be reviewed and edited by peers. The peer-review process did reduce the number of low quality ideas. This process was also rated higher in satisfaction ratings than traditional brainstorming.
\end{abstract}

\section{Introduction}

Brainstorming has been used for years in organizations as a useful way to generate new ideas or perspectives or to develop solutions to existing problems [1,3]. One of the early pioneers of brainstorming, Osborn promoted brainstorming as a way to generate many ideas under the assumption that these ideas would trigger good ideas [5,7]. Through brainstorming many ideas, novel associations and insights can be developed. According to Paulus et al [9], "Brainstorming involves groups of varying sizes that generate as many ideas as possible in a freewheeling, nonevaluative atmosphere", (p.249). They provide further insight into the structure or requirements of successful brainstorming: "Groups are instructed to follow four key rules: generate as many ideas as possible, do not criticize ideas, freewheel by saying all ideas that come to mind, and combine and improve ideas." (p.249-250).

These definitions of brainstorming indicate that the aim is to produce as much content (e.g., brainstorming ideas) as possible; this content will lead to further connections and novel insights. GSS tools were developed to facilitate this brainstorming, further improving the brainstorming experience by adding qualities such as anonymity and parallel brainstorming submissions [11]. Each participant can participate anonymously, allowing each brainstorming idea to be evaluated on its own merits rather than who suggested the idea. Perhaps more importantly, GSS tools allow the participants of a brainstorming session to all brainstorm at the same time. All of the participants can be entering brainstorming ideas at the same time instead of taking sequential turns talking and submitting ideas.

Traditional brainstorming using a GSS tool enables the participants to enter brainstorming ideas at will. Based on the ease of use of the GSS software and the goals of the brainstorming session (i.e., generate as many ideas as possible), the level of quality of the comments varies dramatically [13]. The brainstorming input may be low quality for various reasons: grammatical errors, ambiguous statements, unclear references, incomplete ideas, etc [15].

These low quality ideas cause problems for the group. First, these comments are harder to parse and internalize. Second, the comments require more time, adding to the potential of saturating the group's processing bandwidth. This becomes especially true when the collaborative group increases in size to potentially very large groups. These groups can generate 500-1000 lines of input in one hour [16]. This volume of input may overwhelm both the facilitators and the participants.

This research examines a potential way to improve the quality of the brainstorming ideas that are generated by the group. This particular type of brainstorming would be useful in collaborative sessions with large groups. In large group brainstorming, the group has enough human resources whereby many ideas can be generated. The goal of this type of brainstorming is a subtle shift from the traditional approach of generating as much content as you can to trying to produce a more cohesive and higher quality set of brainstorming ideas.

\section{Peer-reviewed brainstorming}

Peer-reviewed brainstorming is a different approach to brainstorming that requires each brainstorming idea 
to be edited by a predetermined number of anonymous peers [15]. The original brainstorming submission is submitted to a review queue where it is randomly assigned to anonymous peers. Each peer is able to view the original comment and is prompted to improve upon the original idea (see Figure 1 in Appendix A). After the peer reviews are complete, the original comment plus the peer-reviewed versions of the comment are submitted to yet another random and anonymous peer for selection of the "best" version. Figure 2 in Appendix A shows the interface for this selection.

One of the main objectives of this approach is to limit the volume of noisy comments that are submitted to the system. Noisy comments are defined as comments that are either off-topic, non-solutions, or duplicates. It is posited that the peer-review process will reduce the likelihood that participants submit noisy comments and that the peer review process will ultimately improve the overall quality of the brainstorming ideas; participants may be less likely to invest time generating noisy comments if it is known that all comments will be peer reviewed.

This approach exemplifies the idea that more brainstorming comments are not always better; some research has called into question the notion that quality is a function of quantity $[17,18]$.

One potential benefit of the proposed brainstorming process is that by forcing a predetermined number of peers to edit each original brainstorming comment, the system is essentially forcing those peers to more fully process and synthesize the idea, resulting in a deeper understanding of the original comment and its associated issues. The end result may be better comprehension of the issues and suggestions [15].

\section{Methodology}

\subsection{Research questions}

The objective of this research experiment is to further understand the impact of the peer-review process on the group as compared with traditional, free brainstorming. This research project is a pilot study that is exploratory in nature due to the lack of existing literature on the impact of peer-reviewed brainstorming. As such, several research questions are investigated:

1. Does the peer-review process impact the quantity of noise?

The act of reviewing each brainstorming idea may limit the quantity of noise generated since the participants know that each idea will be reviewed. The knowledge that each idea will be reviewed may deter participants from entering noisy input.
Alternatively, the process may not change the likelihood of participants entering in non-substantive ideas; the participants may feel undeterred in their desire to submit ideas that are noisy.

2. Does the peer-review process impact the quality of the ideas generated?

3. What is the impact on the number of valid ideas generated?

The objective of many brainstorming sessions is to generate as many ideas as possible, realizing that many of these ideas will not be feasible or particularly valuable. These types of sessions value broad thinking that may yield novel insights or approaches or may trigger new insights in the group. One potential negative outcome with the peer-review process is that it may stifle this productivity; the group may not be as prolific in generating a wide variety of ideas. The potential of having a narrower set of brainstorming ideas could limit the creative thinking and potentially the quantity of valid ideas.

4. What is the impact on participant's satisfaction levels with the process and the results?

One other potential risk of the peer-review process is that it might be too onerous for the participants. The review process itself might be perceived as too much work or too much overhead, negatively impacting perceptions of satisfaction. This negative perception could impact ratings of satisfaction with the process as well as the final product.

\subsection{Experimental setup}

A pilot study was conducted to examine these research questions and to inform future experimental design. Subjects were randomly assigned to one of two treatment groups during participant check in; five groups participated in each treatment condition. Both treatment groups utilized a Group Support System (GSS) to brainstorm and then rate the brainstorming ideas for a period of 20 minutes. The brainstorming topic was a closed-ended, hidden profile task about a school of business that is facing various challenges [2]. The participants were asked to brainstorm recommendations to improve the situation at the school.

\subsection{Independent variable}

The two treatments consisted of a control and a treatment condition. The control condition was a traditional brainstorm session where the participants 
were able to brainstorm freely without any constraints or reviews. The participants are able to submit any comments, critiques, or off-topic suggestions.

The treatment condition required the participants to use a peer-reviewed brainstorming process. Each brainstorming idea had to be peer reviewed before it would be shown to the entire group. Each brainstorming idea was subjected to two random peers that would edit, modify, or create their own versions of the original comment. The resulting three comments, the original plus the two peer-generated versions, were then submitted to another random peer for selection of the "best" alternative. This means that for every idea that is in the brainstorming idea pool and shown to the entire group, four people were involved in the process. The system made sure that all roles were random and that no one person could perform more than one of the roles for a given idea. Appendix A contains two screenshots showing the edit interface and the selection of the best option interface. It should be noted that all of this activity was conducted anonymously.

The subjects worked together in groups of 6-7 to brainstorm solutions to fix the school of business. The average size of the group was 6.7. The size of the groups was selected because it represents approximately the smallest size group that can successfully execute the peer-review process since each brainstorming idea must be evaluated by two peers and the best version selected by a third. Groups smaller than six may not provide the requisite human capital to enable successful brainstorming. As such, this group size represents a logical starting place for a pilot study with successive experiments increasing the size of the collaborative group.

The participants were instructed to also provide quality ratings for each brainstorming idea. The participants were free to evaluate the ideas at any time during the 20-minute session. The subjects were given alerts when there were five minutes remaining and one minute remaining.

At the conclusion of the brainstorming session, the participants each completed a questionnaire regarding their experiences. The specific questions were derived from previous literature on brainstorming and GSS usage and will be discussed in the next section $[4,6,8]$.

\subsection{Dependent variables}

Several self-reported measures were gathered via the post-survey. These perceptual measures included the following:

- How satisfied with the process How satisfied with the results

- How enjoyable

\section{- How motivated}

Quality measures for each brainstorming idea were assessed by each participant. Each brainstorming idea was evaluated on two different dimensions: creativity and feasibility. Creativity refers to the degree to which the idea is novel or original and feasibility refers to how easily the idea can be implemented [10]. These ratings are not all encompassing as there are many dimensions that could be used. These dimensions were based on previous literature regarding brainstorming quality [12].

The participants assessed the quality ratings on Likert-style scales from 1(low creativity, low feasibility) to 5 (high creativity, high feasibility) for both of the dimensions.

The number of valid ideas for each group was also generated. Valid ideas are defined as ideas that are ontopic and are not duplicates. All off-topic, non-solution, and duplicate comments were removed from the data set. The result was a clean, on-topic, and original comments. Off-topic comments included any comments that do not relate to the task (e.g., "I'm tired of brainstorming"). Non-solution topics include comments that relate to the task but do not present a solution (e.g., "What kind of incentives? It talks about budget cuts..."). Duplicate ideas refer to the number of comments that replicate an idea that was already presented.

\subsection{Participants}

Subjects for this experiment were recruited from an honors section of an introductory Management Information Systems course. The subjects were given course credit for participating in the experiment. Sixtyseven subjects participated.

\section{Results}

The first analysis investigated the impact of the peerreview process on the quantity of noise. This analysis was conducted by comparing the number of off-topic, non-solution, and duplicate comments for each group. An independent samples t-test was performed to compare the two treatments. The results show a near significant difference between the control and treatment groups $t(4)=1.853, p=0.068$ (one-tailed). The control condition had a mean number of invalid ideas of 20.4 (standard deviation $=20.562$ ) and the treatment condition had a mean number of invalid ideas of 3.2 (standard deviation $=2.863$ ). The variances failed the Levene's test of equality; equal variances were not assumed.

The lack of significance on this particular analysis may be due to several factors. First, a twenty-minute 
brainstorming session may not be long enough to see the full benefit of the peer-review approach. It is possible that the time period was too create an environment where non-solutions become more prevalent. Second, the limited sample size may mask some of the effect of the treatment. While not significant, these results are promising.

The second analysis investigated the mean quality of the ideas generated. Independent samples t-tests were performed to compare the control and treatment conditions along the creativity and feasibility dimensions. These two dimensions included values from 1 (low creativity, low feasibility) to 5 (high creativity, high feasibility). The mean values for these conditions are presented in Table 1.

Table 1. Mean creativity and feasibility ratings by condition

\begin{tabular}{|ll|r|r|}
\hline & Condition & \multicolumn{1}{c|}{ Mean } & Std. Dev \\
\hline \multirow{3}{*}{ Creativity } & Control & 3.572 & .155 \\
& Treatment & 3.772 & .237 \\
& Control & 3.680 & .072 \\
Feasibility & Treatment & 3.780 & .070 \\
& & &
\end{tabular}

There was no significant difference between the control and treatment conditions for the average creativity rating, $t(8)=-1.572, p=0.077$ (one-tailed). The extra work of the peer-review process did not negatively impact the average rating of creativity as compared with the control condition.

Looking at the feasibility ratings, the treatment condition produced a significantly higher average feasibility rating as compared with the control condition, $t(8)=-2.219, p<0.05$ (one-tailed).

The next set of analyses compared the number of high quality ideas and low quality ideas by treatments. High quality ideas are defined as comments that receive a composite rating of 3.5 or higher on a scale of 1-5. Table 2 shows the mean number of highly creative and highly feasible ideas by treatment.

Table 2. Mean number of highly creative and highly feasible ideas by treatment

\begin{tabular}{|ll|r|r|}
\hline & Condition & \multicolumn{1}{c|}{ Mean } & Std. Dev \\
\hline \multirow{2}{*}{ High Creative } & Control & 13.40 & 4.159 \\
& Treatment & 10.40 & 3.435 \\
High Feasible & Control & 15.20 & 1.924 \\
& Treatment & 10.00 & 3.162 \\
\hline
\end{tabular}

The control condition produced significantly more highly feasible ideas than the treatment condition, $t(8)=$ $3.141, p<0.05$. No significant difference was found in the number of highly creative ideas between the control and treatment conditions, $t(8)=1.244, p=0.249$. The peer-review process did not significantly alter the ability to the groups to produce highly creative ideas.

The number of low quality ideas was compared using independent sample t-tests. Low quality ideas are defined as comments that receive a composite rating of 3.0 or lower on a scale of 1-5. Table 3 shows the mean and standard deviation of the number of low creativity and low feasibility ideas by treatment.

Table 3. Mean number of low creativity and low feasibility ideas by treatment

\begin{tabular}{|ll|r|r|}
\hline & Condition & Mean & Std. Dev \\
\hline \multirow{2}{*}{ Low Creativity } & Control & 2.60 & 1.517 \\
& Treatment & .40 & .894 \\
& Control & 2.00 & .707 \\
Low Feasibility & Treatment & .40 & .548 \\
\hline
\end{tabular}

The control condition produced significantly more low feasibility and low creativity ideas than the treatment condition, $t(8)=4.000, p<0.05$ and $t(8)=$ 2.794, $p<0.05$, respectively The peer-review process significantly reduced the number of low quality brainstorming ideas.

The next analysis looked at the quantity of valid ideas generated during the brainstorming session. Overall, the control condition generated $61 \%$ valid ideas. The treatment condition generated $82 \%$ valid ideas. Table 4 presents the mean and standard deviation for the total number of comments and the number of valid comments per condition.

Table 4. Mean number of comments and valid comments by treatment

\begin{tabular}{|l|l|r|r|}
\hline & Condition & \multicolumn{1}{|c|}{ Mean } & Std. Dev \\
\hline \multirow{3}{*}{ Total Comments } & Control & 42.00 & 20.845 \\
& Treatment & 16.20 & 3.347 \\
& Control & 21.60 & 2.510 \\
Valid Comments & & 13.00 & 2.828 \\
& Treatment & & \\
\hline
\end{tabular}

The control condition yielded significantly more ideas overall than the treatment condition, $t(4)=2.733$, $p<0.05$ (one-tailed), not assuming equal variances. Likewise, the control condition yielded significantly 
more valid ideas than the treatment condition, $t(8)=$ 5.085, $p<0.05$.

The next set of analyses investigated the selfreported measures that were captured during the postsurvey. Summary statistics for satisfaction with the process and satisfaction with the results is presented in Table 5. The ratings for these two measures ranged from 1 (satisfied) to 5 (dissatisfied).

Table 5. Mean process and outcome satisfaction by treatment

\begin{tabular}{|ll|r|r|r|}
\hline & Condition & \multicolumn{1}{c|}{$\mathrm{N}$} & \multicolumn{1}{c|}{ Mean } & Std. Dev \\
\hline \multirow{3}{*}{ Process } & Control & 32 & 2.16 & .954 \\
& Treatment & 35 & 1.69 & .832 \\
& Control & 32 & 2.13 & .942 \\
\multirow{2}{*}{ Outcome } & Treatment & 35 & 1.69 & .993 \\
& & &
\end{tabular}

The participants in the treatment condition rated their satisfaction with the process significantly better than the participants in the control condition, $t(65)=$ 2.156, $p<0.05$. Looking at the satisfaction with the outcome, the treatment condition again produced significantly better ratings than the control condition, $t(65)=1.853, p<0.05$ (one tailed). The peer-reviewed process produced better satisfaction ratings than free brainstorming.

The participants were also asked how enjoyable they found the experience. The scale on this measure was from 1 (not at all enjoyable) to 5 (very enjoyable). Table 6 shows the mean and standard deviation by treatment for this measure.

Table 6. Mean enjoyment rating by treatment

\begin{tabular}{|ll|r|r|r|}
\hline & Condition & \multicolumn{1}{c|}{$\mathrm{N}$} & \multicolumn{1}{c|}{ Mean } & Std. Dev \\
\hline \multirow{2}{*}{ Enjoyable } & Control & 32 & 3.78 & .870 \\
& Treatment & 35 & 3.86 & 1.004 \\
\hline
\end{tabular}

No significant difference was found between the control and the treatment conditions regarding how enjoyable the participants found the experiment, $t(65)=$ $-0.329, p=.743$.

The last post-survey analysis looked at the question that asked the subjects to evaluate how much they participated in the brainstorming session. The scale was 1 (not very much) to 5 (a great deal). Table 7 shows the mean and standard deviation by treatment.
Table 7. Mean participation level by treatment

\begin{tabular}{|ll|r|r|r|}
\hline & Condition & \multicolumn{1}{c|}{ N } & Mean & Std. Dev \\
\hline \multirow{3}{*}{ Participation } & Control & 32 & 3.91 & .641 \\
& Treatment & 35 & 4.26 & .852 \\
\hline
\end{tabular}

Participants in the treatment condition felt they participated significantly more than the participants in the control condition, $t(65)=-1.891, p<.05$ (single tail). By engaging in the peer-review process, the participants felt they participated more than the control group. Participation may play a role in keeping participants active in the collaborative activities.

One of the potential concerns with peer-reviewed brainstorming is that the length of time between when an idea is originally typed and subsequently viewed by the entire group is considerably longer than in a free brainstorming scenario. This lag between initial idea conception and consumption by the entire group may lead to more duplicate ideas being generated. The mean and standard deviation are shown in Table 8.

Table 8. Mean number of duplicates by

\begin{tabular}{|l|l|r|r|}
\hline \multicolumn{1}{|c|}{ treatment } \\
\hline \multirow{3}{*}{ Duplicates } & Condition & \multicolumn{1}{c|}{ Mean } & \multicolumn{1}{c|}{ Std. Dev } \\
& Control & 4.40 & 2.408 \\
& Treatment & 2.60 & 2.881 \\
\hline
\end{tabular}

No significant difference was found in the number of duplicate comments between the two treatment conditions, $t(8)=1.072, p=.315$. The increased delay in total submission time did not yield a higher number of duplicate comments in the treatment condition as compared with the control condition.

Finally, analysis was conducted to examine which version of the submitted comment was selected as the "best". The purpose behind this investigation is to see if the peer edits were selected as being better than the original or if the original ended up being selected more than either of the two peer edits. It should be noted that versions two and three occur in parallel and independently. Version three is not able to see version two. The original version was selected as the best $31 \%$ of the time. Version two was selected $23 \%$ of the time and version three was selected $46 \%$ of the time. This distribution indicates that the peers who authored the second and third versions of the original comment were 
able to improve upon the original comment, which is one of the main objectives of peer-reviewed brainstorming.

\section{Discussion and future directions}

The results from the peer-review brainstorming pilot study provide interesting many interesting insights into the potential of this new brainstorming technique. These early results indicate that additional, more thorough experimentation is warranted to further understand the positive and negative implications of utilizing a review process in the brainstorming process.

The first set of data analysis procedures investigated the quantitative data that was gathered from the GSS brainstorming tool. These analyses looked at things like the number of non-solution comments and the number of high quality comments. These quantitative results yield some positive and some negative impacts of peerreviewed brainstorming.

One of the major theorized benefits of this brainstorming approach is that fewer noisy comments will be generated by the group. The review process is intended to reduce the probability of individual participants entering in noisy comments. However, the results do not line up with this expectation. No significant difference was found between the conditions when looking at the number of non-solution, off-topic, and duplicate comments.

Looking at these results, several plausible explanations exist. First, as mentioned previously, the participants were only allowed to brainstorm for a twenty-minute period. This time period is relatively short for brainstorming activities and may have been short enough to deter the number of noisy comments. Other research has shown that the number of off-topic and non-solution ideas increases over the duration of the brainstorming period [14]; this time duration may not have been long enough to solicit similar effects. Second, the group size may have also limited the number of noisy comments. The experimental groups were limited to 6-7 participants. This relatively small group provides a different experience than brainstorming in a larger group. In a larger group, brainstorming ideas are constantly being submitted and the user screen refreshes quickly. This volume of ideas being generated may lead to two consequences. First, the participants may spend more time reading the comments from the peers and entering responses to those comments (e.g., non-solution comments). Second, the increased volume may provide an increased impetus for some members of the group to social loaf and submit off-topic comments since it appears that a lot of collaborative work is being done by the group. Additional research in this area could include an investigation regarding the size of the group and the correlation with noisy comments.

The free brainstorming condition produced a significantly higher total number of ideas than the peerreviewed brainstorming condition and a significantly higher number of valid ideas. On the surface, this result appears to be a negative externality of the peer-review process. However, this result may not be negative depending on the context. This brainstorming approach was designed with the goal of facilitating large group collaboration; this approach was designed to reduce the unwieldy volume of comments that comes from increasingly large groups. Ideally, the peer-review process would solely limit the volume of low quality or noisy ideas. However, limiting the overall number of ideas is not necessarily a problem as the large group will be able to generate a high volume of ideas. As seen previously, this approach did lead to $82 \%$ of the comments being valid whereas only $61 \%$ of the free brainstorming ideas were valid. These results are consistent with the aims of peer-reviewed brainstorming.

Further analysis of the quality of the brainstorming ideas yielded interesting results. The peer-review process produced significantly fewer low quality brainstorming ideas. It should be noted that this result could be a function of the overall reduced number of ideas being generated by the treatment groups or by the treatment itself. Similarly, the treatment condition yielded a significantly higher average feasibility rating than the treatment and a nearly significant increase in average creativity ratings. Overall, these quantitative analyses present somewhat of a mixed result for peerreviewed brainstorming. However, there are enough positive results that warrant further investigation into controlling the brainstorming process. One of these positive results is the fact that the original brainstorming idea was selected as the best option only $31 \%$ of the time while one of the edited versions was selected $69 \%$ of the time. The anonymous reviewers were able to improve upon the original comment as judged by a randomly selected peer.

The subjective, perceptual measures gathered during the post-survey provide much more favorable results for peer-review brainstorming. One of the speculated negative impacts of the peer-review treatment is that the review process itself would be too onerous for the participants. The review might require too much cognitive effort and reduce how favorable each participant finds the process. This is a large risk as participant motivation is one of the key drivers of success in GSS efforts.

The post-survey produced some interesting results in this regard. First, the participants were asked to evaluate how enjoyable the experiment was. No significant 
difference was found between the two treatments. In other words, the overhead of the review process did not negatively impact the perceived enjoyment of the process; the review process was as enjoyable as the free brainstorming process.

Second, the participants in the treatment rated their satisfaction with the process significantly higher than those in the control condition. The participants in the treatment condition were also significantly more satisfied with the outcome than those in the control condition. Contrary to the potential risk, the participants were more satisfied in the peer-review brainstorming approach.

Finally, when asked to evaluate how much they participated in the experiment, the treatment participants rated their participation levels as being significantly higher than the control condition participants. Again, the feeling of being involved and participated is a key factor in successful GSS implementation.

The post-survey favorable results for the peerreviewed treatment present one compelling reason to continue research in this area. The logic or reasoning behind the positive ratings could be explained by a few different rationales. First, the peer-reviewed treatment provides more opportunities for the participants to stay active in the brainstorming process. If a particular subject has no more original ideas to contribute, he or she may elect to review and edit the ideas from the peers rather than simply passively reading the ideas of others. Perhaps this increased level of activity produced an increased level of satisfaction with the process. This would also explain the higher levels of participation that are reported by the participants in the treatment condition. Second, the participants may feel like they are contributing more value when they are able to improve upon the ideas of peers. Some individuals may not feel like they are contributing significantly in regards to original brainstorming ideas. However, while these people may not have original ideas, they can still contribute to the group effort by improving upon the original ideas of others.

Future research in this area is currently underway. The next iteration of this experiment will consist of two major changes to the experimental design. First, the duration of the brainstorming session will be increased to 30 minutes. The increased time will accommodate the increased time requirements of the peer-review process. Second, the group size needs to be increased to approximately ten participants. When requiring four of the participants to process any given brainstorming idea, peer-review brainstorming is resource intensive and severely constrains the ability of the group to produce ideas. By increasing the number of participants, the group will have a higher level of throughput and be able to generate ideas more efficiently.

Subsequent iterations of this experiment will investigate the dimension of group size and group synchronicity. The benefits of peer-review brainstorming may not be fully realized until the group size reaches a particularly large group size. The impact of synchronicity on the ability of the group to execute this brainstorming approach also needs to be investigated. It is unknown what the impact of brainstorming in an asynchronous environment might be. Asynchronous brainstorming could lead to a longer duration for the brainstorming period and ultimately a more productive result from peer-reviewed brainstorming. Alternatively, brainstorming asynchronously may decrease the motivation of the participants to review and edit comments from peers; the participants might feel less of a sense of urgency or immediacy and the productivity might be reduced.

\section{Conclusion}

Brainstorming in collaborative GSS sessions is one of the fundamental and foundational activities. Brainstorming is widely used as it is so applicable to a wide variety of contexts and problem solving scenarios. Generating ideas or solutions as a group provides an effective way to develop novel insights and commence the process of identifying solutions or courses of action.

It can be argued that most brainstorming sessions are designed with the goal of identifying as many possible ideas as possible during the allotted time. Brainstorming typically encourages participants to think outside the box in order to develop a wide range of solutions. The expectation is that generating a large volume of ideas will lead to more ideas and ideas that are more creative or novel than previous ideas; brainstorming ideas can trigger new thoughts or connections between concepts or ideas.

Given the goal of harnessing as much brainstorming input as possible, GSS tools and methodologies should focus on reducing or eliminating any stimuli or factors that may hinder the efficiency with which ideas are generated. The brainstorming process should be as easy and low overhead as possible in order to maximize the number of ideas generated.

However, there do exist situations in which the objectives of brainstorming do not include generating as many ideas or as much input as possible. In large group collaboration, the group is able to generate an immense volume of input in a short period of time. Modifications to the brainstorming approach are needed in order to refine the brainstorming and reduce the possibility that the brainstorming input overwhelms the GSS resources, including participants and facilitators; in large group 
settings, the risk of information overload should be explicitly and consistently addressed.

Peer-reviewed brainstorming is one potential modification to traditional brainstorming that attempts to better control or refine the ideation process. Each brainstorming idea is routed to two independent, anonymous, and random peers for editing. The peers are able to read the original brainstorming comment and improve upon the comment. The improvement may be something as simple as grammar or word choice to improve comprehension or it could be a refinement of the idea to be develop a more salient comment.

This modified brainstorming process does reduce the efficiency with which the group develops a set of brainstorming ideas. The review process forces a certain level of overhead or additional work that each of the participants must shoulder. However, the tradeoff between the lack of efficiency is an improved, more cohesive and less noisy, set of brainstorming ideas. In large group collaboration scenarios, this tradeoff may be worthwhile. Large groups can overcome the lack of efficiency with the increased availability of human resources, which in turn places a premium on a higher quality pool of ideas.

The results from this pilot study indicate that this approach may be successful in this regard. The participants did not appear to suffer from the extra burden of the peer-review overhead; the post-survey responses were very favorable. Further research will examine and fine tune this approach in order to optimize the review process.

\section{References}

[1] Grossman, S.R., Rodgers, B.E., and Moore, B.R. Turn group input into stellar output. Working Woman 14, 12 (1989), 36-38.

[2] Mennecke, B.E. and Wheeler, B.C. Tasks matter: modeling group task processes in experimental CSCW research. (1993), 71-80 vol.4.

[3] Grossman, S.R. Brainstorming Updated. Training and development journal 38, (1984), 84-87.

[4] Briggs, R.O. and de Vreede, G.-J. Measuring satisfaction in GSS meetings. ICIS '97: Proceedings of the eighteenth international conference on Informationsystems, Association for Information Systems (1997), 483-484.

[5] Osborn, A.F. Applied Imagination: Principles and procedures of creative problemsolving. 1957. New York: Charles Scribner's Sons, (1957).
[6] Santanen, E.L. Directed brainstorming and the cognitive network model of creativity: An empirical investigation of cognitive factors related to the formation of creative solutions using an electronic brainstorming environment. 2002.

[7] Osborn, A.F. Applied Imagination: Principles and procedures of creative thinking. Scribner's, New York, 1953.

[8] Santanen, E.L., Briggs, R.O., and Vreede de, G.J. Causal relationships in creative problem solving: Comparing facilitation interventions for ideation. Journal of Management Information Systems 20, 4 (2004), 167-198.

[9] Paulus, P.B., Larey, T.S., and Ortega, A.H. Performance and Perceptions of Brainstormers in an Organizational Setting. Basic and Applied Social Psychology 17, 1-2 (1995), 249-265.

[10] Diehl, M. and Stroebe, W. Productivity loss In brainstorming groups : Toward the solution of a riddle. Journal of Personality and Social Psychology 53, 3 (1987), 497-509.

[11] Nunamaker Jr., J.F., Briggs, R.O., Mittleman, D.D., and Nunamaker Jr., J.F. Electronic Meeting Systems: Ten Years of Lessons Learned. In D. Coleman and R. Khanna, eds., Groupware: Technology and Applications. Prentice Hall PTR, Saddle River, NJ, 1995, 149-193.

[12] Barki, H. and Pinsonneault, A. Small group brainstorming and idea quality: Is electronic brainstorming the most effective approach? Small Group Research 32, 2 (2001), 158-205.

[13] Chen, H., Houston, A., Nunamaker Jr., J.F., and Yen, J. Toward intelligent meeting agents. Computer 29, 8 (1996), 62-70.

[14] Helquist, J.H., Santanen, E., and Kruse, J. Participant-driven GSS: Quality of Brainstorming and Allocation of Participant Resources. IEEE, Washington, DC, USA, 2007.

[15] Helquist, J.H., Kruse, J., and Adkins, M. Group support systems for very large groups: A peer review process to filter brainstorming input. Proceedings of the Americas Conference on Information Systems, (2006). 
[16] Chen, H., Hsu, P., Orwig, R., Hoopes, L., and Nunamaker J. F., J. Automatic concept classification of text from electronic meetings. Communications of the ACM 37, 10 (1994), 56-73.

[17] Briggs, R.O., Reinig, B.A., Shepherd, M.M., Yen, J., and Nunamaker Jr., J.F. Quality as a function of quantity in electronic brainstorming. Proceedings of the Thirtieth Hawaii International Conference on Systems Sciences, (1997).
[18] Reinig, B.A. and Briggs, R.O. Measuring the Quality of Ideation Technology and Techniques.

Proceedings of the 39th Annual Hawaii International Conference on System Sciences, (2006).

\title{
8. Appendix A
}

\begin{abstract}
Please technology adaptation. early, middle and those who never adopt new technology. Why do things revise this that are useful not take off even if they are trainedd to use it. How do you get to that critical mass. comment: Does that concept apply to these refugee camps.
\end{abstract}

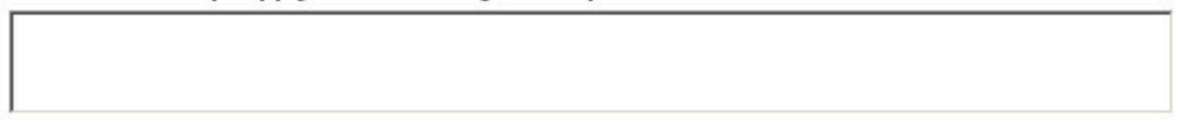

Figure 1. Peer-review edit interface screenshot

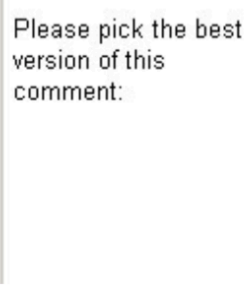

1. technology adaptation. early, middle and those who never adopt new technology. Why do $O$ things that are useful not take off even if they are trainedd to use it. How do you get to that critical mass. Does that concept apply to these refugee camps.

2. How does technology adoption impact the decision to make certain technologies available?

6 3. For available technologies, ensure training and help is available for the refugees so that the assets are utilized

Figure 2. Peer-review select best option screenshot 